\title{
Quality Control of Digitized Data and Inclusion of Essential and Meaningful Checkpoints
}

\author{
FRANS P. G. M. van der LINDEN and RICHARD L. MILLER \\ Center for Human Growth and Development, The University of Michigan, Ann Arbor, \\ Michigan 48104, USA
}

The collection of vast amounts of digitized data is increasing rapidly in biological research and requires organized storage for efficient retrieval. Other problems are editing the data and verification. Preferably, they are done by a computer program that prints out critical values. Basic derived values that present a good overall view of the essential aspects of an investigation can be calculated and printed out. When longitudinally collected records are analyzed, critical serial values can be included in the basic data.

These suggestions will be examined in the method used in a recent study on about 1,000 dental casts. Data were collected by means of a special instrument, the Optocom, which made it possible to obtain accurate three dimensional information directly from plaster casts (VAN DER LINDEN, IADR Program and Abstracts of Papers, No. 286, 1970). In a complete dentition, 360 points are collected by means of Cartesian coordinates.

To systematize information collection and to facilitate data retrieval, a line number was assigned to each tooth. Fourteen line numbers contained data on height recordings. Three lines contained information gathered on rugae and other nondental points. Line 50 was used to repeat some registrations made at the beginning and was a check for machine errors and constancy in positioning of the cast during recording.

To verify the data, the mesiodistal and buc- colingual dimensions of all crowns were calculated and printed. The same was done for the anteroposterior and vertical relationships on both sides between the corresponding first molars, second premolars or deciduous molars, canines, and central incisors. Finally, two measurements were obtained to check the proper orientation and placement of the dental casts in relation to each other. The print-out of the controlling data was organized in 11 groups added directly after line 50 .

On the last of a series of dental casts from the same individual, longitudinally performed checking values were calculated and printed. Preference was given to values that could help to evaluate quickly the most essential and meaningful characteristics of the total series. The teeth present, the arch width between the corresponding buccal teeth. and the arch depth dimensions were calculated and printed. The same was done for the anteroposterior and vertical relationships for five pairs of teeth.

The calculation and printing out of essential and frequently needed information at the end of a series eliminates the repitition of computations. The data structure allows the inclusion of additional lines so that other calculated information, such as yearly incremental values, may also be included.

Additional information available on request to authors.

Received for publication January 15, 1971.

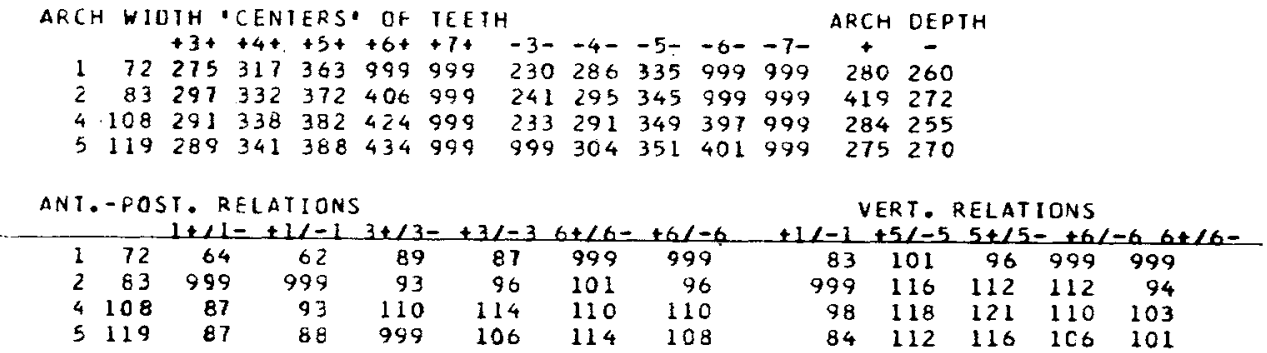

Part of print-out at the end of a series of four sets of dental casts. Age is given in months. 